\section{Distinct potential}

\author{
D. Aled Jeffreys
}

Electroencephalography and Clinical Neurophysiology: Evoked Potential Section. Editor-in-chief Hans van Duijn. Elsevier. 6/yr. \$83 (institutional); $\$ 55$ (individual).

REPORTS of evoked potential studies appear in a wide variety of journals, with the choice of journal usually reflecting the particular aspects of applications being studied. Thus, evoked potential investigations of sensory function are commonly submitted to, say, Experimental Brain Research, Audiology or Vision Research, whereas more psychologically orientated work is likely to be reported in, for example, Psychophysiology. A favoured target for clinically related papers, on the other hand, has long been "The EEG Journal" (Electroencephalography and Clinical Neurophysiology), and it was in response to the steadily increasing proportion of such contributions that this specialized section was introduced in 1984. It is the first publication devoted solely to this topic but, despite the distinct title Evoked Potentials printed on the cover, it remains a constituent part of "The EEG Journal".

According to the introductory editorial, this additional section is devoted to clinical and experimental evoked potential research, but the emphasis is, and probably will continue to be, clinical. In fact, one reason given for maintaining Evoked Potentials as a part of its parent journal was "to demonstrate that the area of evoked potentials is, and should remain, an integral part of clinical neurophysiology". An additional reason for introducing this specialized section was to "increase accessibility to workers in related fields whose interest in clinical neurophysiology is largely confined to evoked potential phenomena". This aim is partially offset, however, because several evoked potential papers continue to appear in the nonspecialized volumes, and the criteria for allocating papers between the different sections are unclear.

The Instructions to Authors are fairly standard. Papers may be in either English or French, but all so far published have been in English: however, abstracts must be submitted in both languages. Both long and short articles are accepted, and they must be accounts of original investigations. There are occasional book reviews and regular advertisements for equipment, but, in general, no editorials, review articles or comments on previous papers. The journal is well produced on good quality, but uncomfortably glossy, paper. The delay from acceptance to publication of most long papers is 6-7 months, and for short communications about 4 months.

Even though the proportion of evoked potential papers in "The EEG Journal" continues to rise, there is no firm evidence that the introduction of Evoked Potentials has as yet either extended the scope of its coverage or diverted many papers from going to other less-specialized journals. Nevertheless, this new section provides a convenient focus for clinically orientated evoked potential papers and will probably continue to gain in importance in relation to its companion volumes.

D. Aled Jeffreys is Senior Research Fellow in the Department of Communication and Neuroscience, Keele University, Staffordshire ST5 5BG. UK

\section{Putting the heat on}

\section{Paul Carnochan}

International Journal of Hyperthermia. Edited by S.B. Field, J. Overgaard, G. Hahn and T. Sugahara. Taylor \& Francis. $6 / y r . £ 80, \$ 144$.

IN RECENT years, the investigation of hyperthermia for the treatment of cancer has graduated from an empirical and largely anecdotal subject to one supported by a growing volume of experimental data. In common with many new branches of science "hyperthermic oncology" draws upon the resources of a wide range of disciplines, creating difficulties both in the communication of new ideas and in gaining efficient access to the relevant literature. The emergence of the International
Journal of Hyperthermia is therefore appropriate. The journal has a truly international character, with an editorial board representing 14 countries and, thus far, a clearly non-parochial content.

The editors have aimed to attract everyone with a serious interest in the subject, and the variety of contributions to date suggests that every effort is being made to maintain a wide readership. The four issues available for review are of a uniformly high standard, showing a good balance between invited review articles and original research papers, and covering the whole spectrum of relevant topics from the subtleties of heat-shock protein synthesis through to the clinical front line. I hope that this diversity in subject matter will be sustained and that, in-time, proportionaily more clinical studies will be represented, reflecting what should surely be the ultimate goal of research effort in this field.

A notably short publication delay of 2-3 months appears to be the norm following acceptance of submitted material, with roughly the same time being taken for refereeing and revision. Overall production standards are high and the choice of a small format with full-width illustrations has ensured excellent clarity.

The journal's long-term prospects will depend upon either the clinical success of hyperthermia in cancer therapy or the editors' ability to attract material from the wider field of related medical topics. Judged purely on the quality displayed so far, however, it clearly deserves to succeed.

Paul Carnochan is at the Institute of Cancer Research and Royal Marsden Hospital, Fulham Road, London SW3 6JJ, UK.

\section{Choice of delivery}

\section{Malcolm Moore}

Journal of Controlled Release. Editors Jan Feijen and Jorge Heller. Elsevier. 9/yr in 3 volumes. $D$ fl. 750 .

Journal of Microencapsulation. Editor J.R. Nixon. Taylor \& Francis. 4/yr. $£ 50, \$ 90$.

THESE two journals have a lot in common. Both were founded in 1984, have international editorial boards and are published in English; in both, the United States is the most frequent, but not predominant, source of contributions. Their editorial policies imply a greater breadth of coverage than is evident in recent issues, but in this respect editors are at the mercy of contributors and inevitably papers from currently active areas of research are in the majority. At the moment, their coverage overlaps significantly.

Journal of Controlled Release deals with the control of the rate or site of release of active agents by any means - chemical, physical or mechanical. Nearly all of the contributions are pharmaceutically orientated, and over half of them are concerned with the preparation or properties of gel or polymeric matrices, ranging in size from microspheres upwards, and their ability to entrap drugs and release them by diffusion or after erosion. Others deal with drug transport across skin, degradable drugpolymer conjugates and emulsion systems. It is disappointing that only one among them is specifically concerned with targeting.

Papers are generally substantial in length and of good quality. Acceptance usually takes several months, and is often granted only after revision which suggests a Rhadamanthine editorial board. Besides full papers there is news from the Controlled Release Society, of which this is the official journal, a calendar of relevant meetings, a review of US patents and an occasional section of book reviews. The quality of print is good, though the 